\title{
Bacteriostatic Substrate by Conductivity Method and Electric Spark Discharge Method Combined with Electrospinning for Silver Dressing
}

\author{
Kuo-Hsiung Tseng, Chaur-Yang Chang, Meng-Yun Chung, and Ya-Lan Tang \\ Department of Electrical Engineering, National Taipei University of Technology, Taipei, Taiwan \\ Correspondence should be addressed to Kuo-Hsiung Tseng; khtseng@ee.ntut.edu.tw
}

Received 30 May 2016; Revised 15 August 2016; Accepted 31 August 2016

Academic Editor: Ming-Guo Ma

Copyright ( $\odot 2016$ Kuo-Hsiung Tseng et al. This is an open access article distributed under the Creative Commons Attribution License, which permits unrestricted use, distribution, and reproduction in any medium, provided the original work is properly cited.

\begin{abstract}
This study uses the conductivity method, Electric Spark Discharge Method, and the electrospinning technique to develop a better silver-based antibacterial agent. The preparation process is free of chemical substances and also conforms to the green energysaving process. The silver iodide was prepared in an iodine agar medium by using the conductivity method. Multiple bacteriostasis experiments showed that the molds grew in the position with iodine of the culture medium after 6 days, as well as in the position with silver iodide after 10 days. The results prove that silver iodide has better bacteriostatic ability than povidone iodine. The nanosilver colloid was prepared in the PVA solution by using the Electric Spark Discharge Method. UV-Vis, Zetasizer, and SEM-EDX analyses proved that the PVA solution contained nanosilver colloid with good suspension stability. Finally, the electrospinning technique was used to spin the PVA solution with nanosilver colloid into the PVA nanofibrous membrane. According to UV-Vis analysis, the absorption peak of this nanofibrous membrane is about $415 \mathrm{~nm}$, meaning this nanofibrous membrane contains nucleate nanosilver colloid, and is very suitable for antiseptic dressing.
\end{abstract}

\section{Introduction}

Ever since British researcher Alexander Fleming published his paper on penicillin with bactericidal action in 1929, biomedical circles have put great efforts into the research and development of different antibiotics in order to resist different bacteria; thus, the history of human antibacterial reaches a new milestone, prolonging the average life. Although antibiotics have strong sterilizing effect, if they are applied improperly and fail to completely kill bacteria, then the surviving bacteria evolve into drug-fast variant bacteria by gene mutation. When antibiotics lose their effect on bacteria, infectious diseases are more easily spread as the transport velocity increases, making it more difficult for humans to fight infectious diseases $[1,2]$. Therefore, solving resistance to antibiotics and seeking other methods to kill infectious bacteria have become important topics of medical technology in this century.

Skin is the protection layer for isolating bacteria. When the skin is hurt, the dressing plays an important role of wound care. The method of open dressing is used in the danger of infection or the tissue fluid is not stopping. Normally, when the tissue fluid is still flowing or the wound is not clean, the bacteriostasis is to avoid the deterioration of the wound and the sterilization to heal it. Moreover, if a suitable moist environment is provided, it will accelerate wound healing. To meet the needs of the aforementioned wound care, the development of the dressing, medicine, and assisted therapy is much more improved, such as silver ion, nanosilver particles, nanofibrous membrane, and iontophoretic transdermal drug delivery system.

Silver was actually extensively used as an antibiotic material before antibiotics were invented. However, after the extensive development of antibiotics, the antibacterial role of silver was gradually neglected. In recent years, due to the rapid development of nanotechnology and bacterial resistance, research has again paid attention to silver and silver nanoparticles in biomedical materials [3-5]. Therefore, the development of silver dressing has become a very important part of wound care. At present, silver-bearing dressing 
has been extensively used to treat chronic wounds, as the dressing releases silver ions to control bacteria [6-8]. In addition, because the sterilization of silver is physical, there will not be any drug resistance problem [9]. Among the silver dressing preparation methods, the physical method has cleaner silver particles than the chemical method. Therefore, this paper uses the conductivity method, Electric Spark Discharge Method, and the electrospinning technique to research a better silver-based antibacterial agent.

There have been many studies regarding electrospinning, but also a few studies that mix PVA with collagen. For example, the nanofibrous membrane of PVA can remedy the defects of traditional nonwoven fabric [10]. According to FTIR analysis, spun yarn contains collagen. In terms of antibacteria, some studies employed chitosan as the antibacterial material to successfully spin biodegradable dressing [11]. Other studies used the electrospinning technique to jet collagen nanofiber, where the mean size of the fiber was about $100 \mathrm{~nm}$, and examined collagen spinning for fibroblast culture in vitro [12].

\section{Material and Methods}

In recent years, many nanometals have been developed and applied to medical products. The nanometal preparation methods encompass physical, chemical, and mechanical. Many studies have used ESDM to prepare nanometal, which is a preparation method that takes the principle of a physical electrical spark discharge [13-15]. In comparison to the mechanical and chemical methods, the nanometal preparation process by ESDM is free from pollution, and the nanoparticles are easily collected.

2.1. Principle of Preparing Nanosilver Colloid by ESDM. EDM is a hot working method that converts electrical energy to thermal energy in order to quickly melt a workpiece. The method of using EDM to melt the metal surface to obtain nanometal colloid is called the Electric Spark Discharge Method (ESDM) [16-18]. Figure 1 shows the process of preparing nanometal colloid by ESDM, where $d_{\text {gap }}$ is the distance between two electrodes. Here, $d_{\min }$ and $d_{\max }$ are defined as the minimum distance and maximum distance of the electrode gap, respectively, when the EDM discharge succeeds.

(1) When $d_{\text {gap }}>d_{\text {max }}$, the dielectric fluid has high resistance; thus, the discharge circuit forms an open circuit, as shown in Figure 1(a).

(2) When $d_{\text {min }} \leqq d_{\text {gap }} \leqq d_{\text {max }}$, the electric field intensity is enough to make the dielectric fluid have slight resistivity; thus, an arc is generated between the two electrodes. The electrons quickly impact the upper electrode (anode) in the discharge process, and the cations flow towards the cathode slowly, as shown in Figure 1(b).

(3) In the spark discharge process, the arc temperature is enough to melt the electrode material nearby the arc and gasify the dielectric fluid in the electrode gap.
TABLE 1: Nanosilver colloid preparation parameters.

\begin{tabular}{lc}
\hline Discharge pulse $\left(T_{\mathrm{ON}}-T_{\mathrm{OFF}}\right)$ & $20-10 \mu \mathrm{s}$ \\
Current setting $(\mathrm{IP})$ & $1-1.4 \mathrm{~A}$ \\
Capacitor & $\mathrm{No}$ \\
DI water & $40 \mathrm{~mL}$ \\
Metal wire size & $1 \mathrm{~mm}$ \\
Pressure & $1 \mathrm{~atm}$ \\
SERVO. & $1 / 2$ \\
SENS. & $1 / 2$ \\
$z$-axis & $1 / 2$ \\
Discharge time & $60 \mathrm{sec}$ \\
Metal purity & $99.99 \%$ \\
Temperature & $25^{\circ} \mathrm{C}$ \\
\hline
\end{tabular}

The pressure generated by the gasification expansion of the dielectric fluid compels the molten metal to scatter in the dielectric fluid. The fine particles sputtered in the dielectric fluid are rapidly cooled and condensed by the peripheral cold dielectric fluid to nanoparticles, as shown in Figure 1(c).

(4) When discharge is complete, the dielectric fluid reverts to the state of insulation, as shown in Figure $1(\mathrm{~d})$.

The nanometal colloid can be prepared by ESDM through the aforesaid process. As the electrode gap is the main factor influencing the process, the interelectrode distance shall be readjusted by the servo control system [19].

Figure 2 shows the architecture of preparing nanometal colloid by EDM. In this paper, the upper electrode and lower electrode are made of silver. The charging/discharging circuit provides the impulse voltage required when the electrode is discharging. The servo control system controls the electrode gap, as it judges the interelectrode distance according to the feedback signal of the electrode current, and the driving system and motor readjust the distance between the working electrodes ( $z$-axis); thus, the electrode gap maintains a good discharge interval and the success rate of discharge rises. When the electrode gap is $10 \sim 100 \mu \mathrm{m}$, two electrodes successfully generate arc discharge. The electrode surface is turned molten by the arc temperature and rapidly scattered in the dielectric fluid, which is then cooled and condensed by dielectric fluid to silver nanoparticles [20, 21].

ESDM is used to prepare nanosilver colloid in this paper. The process is characterized by simple and rapid preparation and mass production and is free from any chemical substances; thus, it is a method conforming to green energy-saving process. Table 1 lists the process parameters. The upper and lower electrodes are made of metal silver, with a diameter of $1 \mathrm{~mm}$ and purity approaching $99.99 \%$. The preparation environment is the standard condition, the $T_{\mathrm{ON}}-T_{\mathrm{OFF}}$ parameter of the discharge circuit is set as $20-10 \mu \mathrm{s}$, the beaker capacity is $40 \mathrm{~mL}$, SERVO., SENS., and $z$-axis parameters are set as $1 / 2$, and the dielectric fluid is deionized water (DI water). 


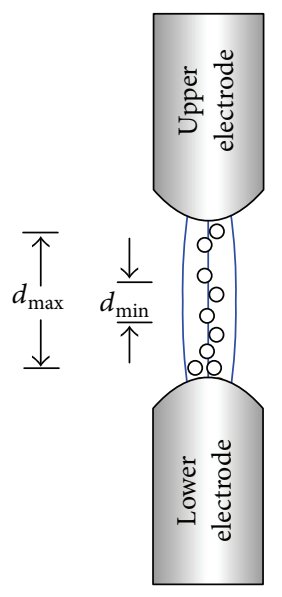

O Electron

- Positive ion

- Metal particle

(a)

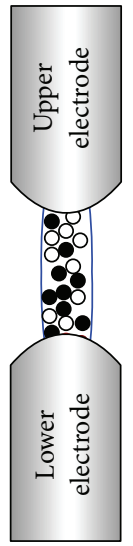

O Electron

- Positive ion

- Metal particle

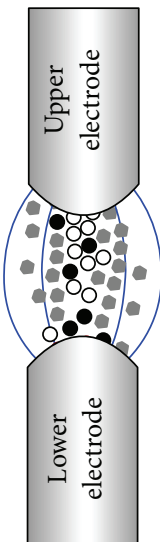

Electron

- Positive ion

- Metal particle

(c)

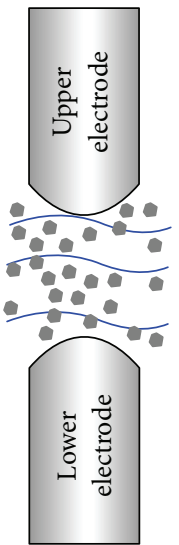

○ Electron

- Positive ion

- Metal particle

(d)

FIGURE 1: Process of preparing nanometal colloid by ESDM.

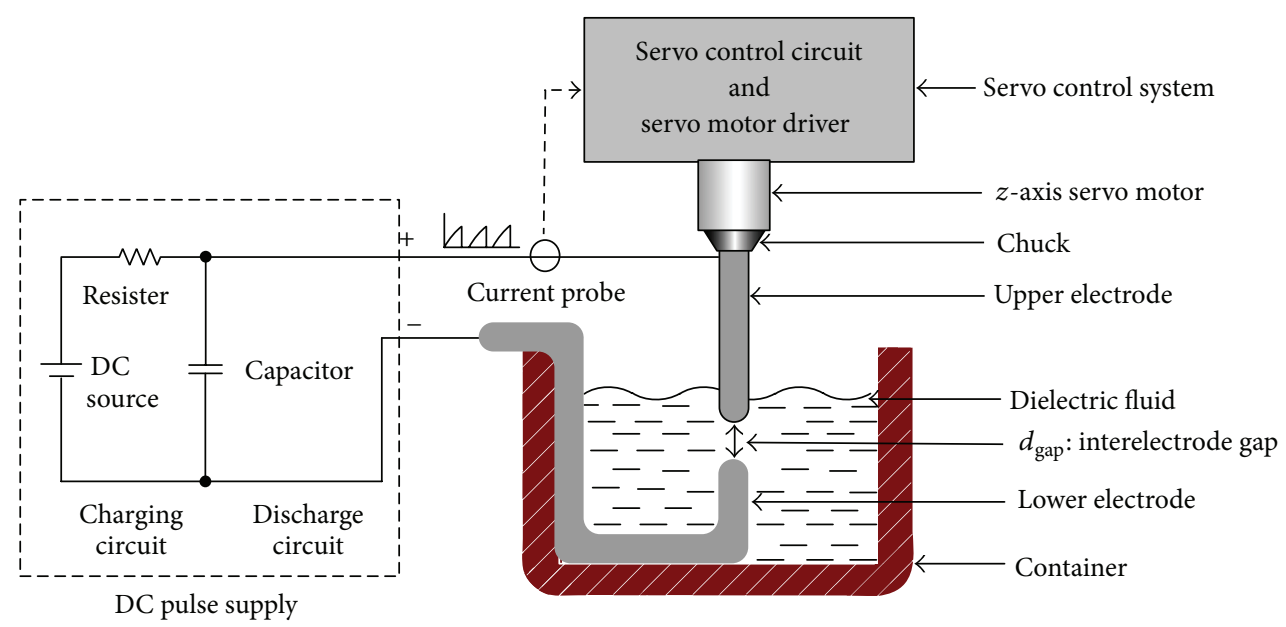

FIgURE 2: Architecture of preparing nanometal colloid by EDM.

In terms of the physical and chemical properties of the nanosilver colloid in this paper, the absorption spectrum, suspension stability, particle size, and composition of the ESDM prepared nanosilver colloid are analyzed by UVVisible Spectroscopy (UV-Vis), Zetasizer, and SEM-EDX. The UV-Vis analysis results show that there is an obvious absorbance peak at $410 \mathrm{~nm}$, as shown in Figure 3(a). The Zetasizer analysis results show that the average Zeta-Potential is about $-39.3 \mathrm{mV}$, meaning the nanosilver colloid has good suspension stability, as shown in Figure 3(b). The SEM analysis results show that the particle size of nanosilver colloid is about $20 \mathrm{~nm}$, as shown in Figure 3(c). The EDX analysis results show that the principal component of nanosilver colloid is silver, and carbon and oxygen are provided by water and the karbogel, respectively, for analysis, as shown in Figure 3(d).
2.2. Preparation of Silver Iodide by Conductivity Method. Povidone iodine is extensively used as a disinfectant, as it slowly releases iodine on the wound tissue for bacteriostasis. As silver has good bacteriostatic ability, if iodine is replaced by silver iodide, then the bacteriostatic effect can be enhanced. This paper uses the conductivity method to prepare silver iodide and examines the bacteriostatic ability of silver iodide according to the fungal growth in the bacteria culture medium.

The preparation of silver iodide by using the conductivity method is by combining the silver and iodine ions in the iodine agar medium into a silver iodide under the electric field effect. Regarding the production of the iodine agar medium, $192 \mathrm{~g}$ water and $8 \mathrm{~g}$ advanced agar powder are prepared in an agar solution at a concentration of $4 \%$. The solution is stirred and heated to $90^{\circ} \mathrm{C}$, and the agar solution is 


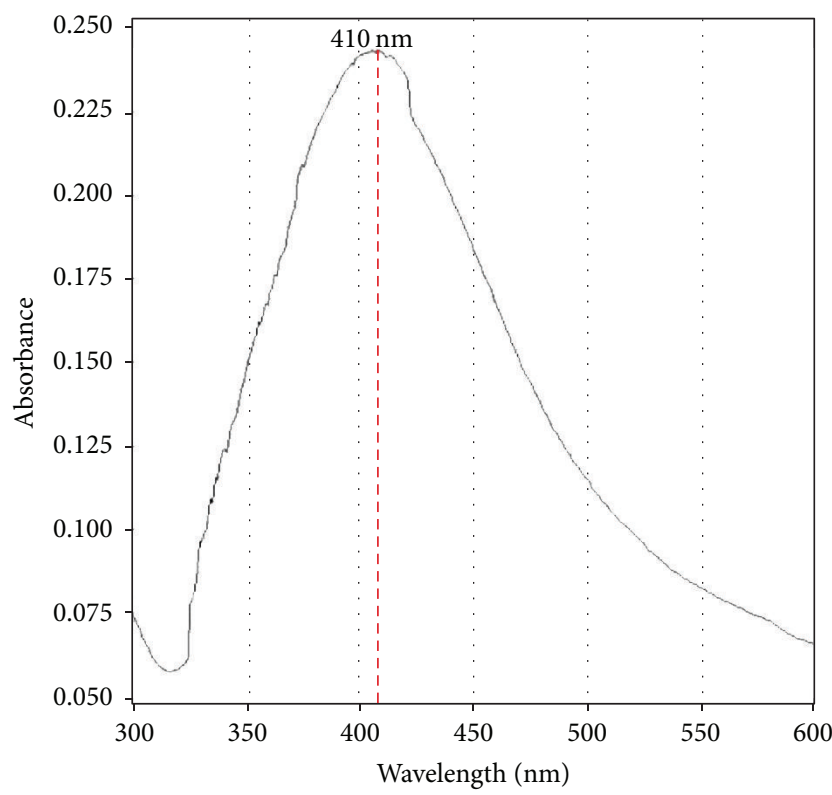

(a)

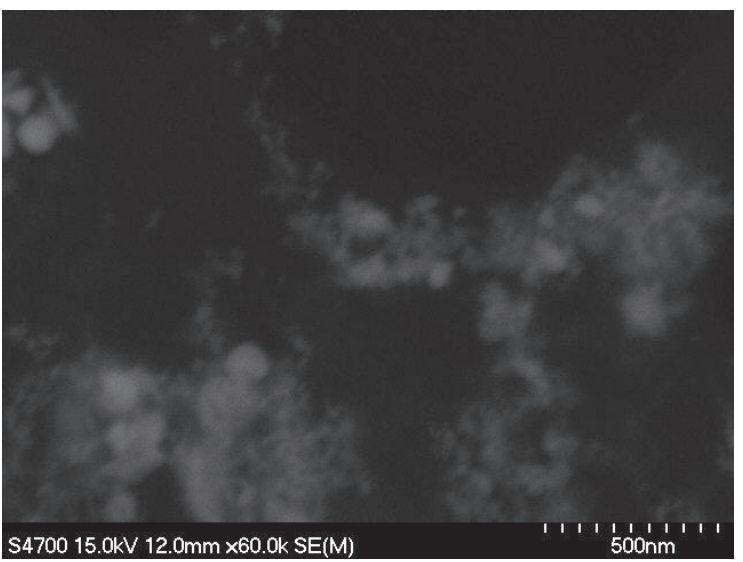

(c)

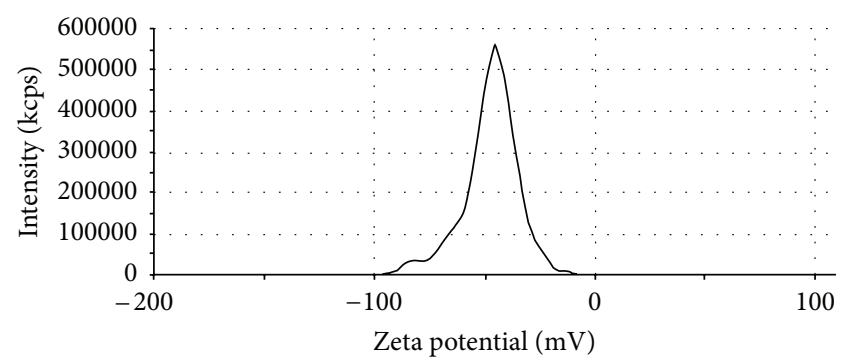

(b)

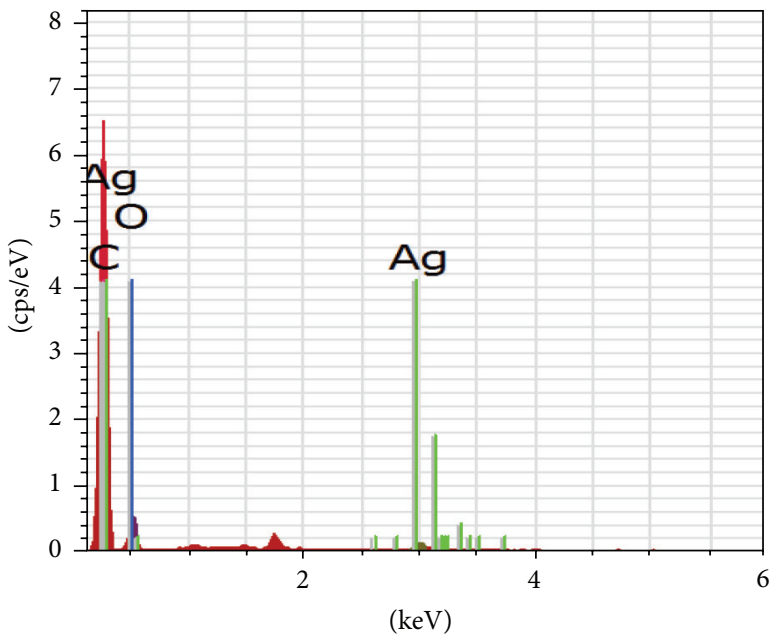

(d)

FIGURE 3: Nanosilver colloid (a) UV-Vis analysis, (b) Zetasizer analysis, (c) SEM image, and (d) EDX component analysis.

mixed with 4 c.c. povidone iodine and poured into the culture dish. The solution is cooled and solidified into an iodine agar medium. As the agar powder contains starch, the iodine agar medium is dark blue.

In terms of preparing silver iodide by the conductivity method, a silver wire and a silver sheet are inserted in the iodine agar medium as electrodes, the anode of the DC voltage source is connected to the silver sheet, and the cathode is connected to the silver wire; thus, the interelectrode current is maintained at $10 \mathrm{~mA}$ within 10 minutes, as shown in Figure 4. In the conductance process, the silver ions at the anode are combined with the electronegative iodine ions to form a light yellow silver iodide. The aforesaid silver wire has a diameter of $1 \mathrm{~mm}$, and the silver sheet is $1 \mathrm{~mm}$ thick, $1 \mathrm{~cm}$ long, and $1 \mathrm{~cm}$ wide.
2.3. Combine ESDM with Electrospinning to Prepare Silver Nanoparticle PVA Nanofibrous Membrane. Traditional textile technology uses melt spinning, dry spinning, or gel spinning to weave fiber, with a diameter of $2 \mu \mathrm{m} \sim 100 \mu \mathrm{m}$, and its size is a micrometer scale. The fabric woven by the electrospinning technique has a nanoscale diameter, higher porosity, and larger surface area, and the pore size is smaller than that woven by the traditional technique. Therefore, the nanofibrous membrane made by the electrospinning technique has high toughness and tear resistance.

A schematic diagram of electrospinning is shown in Figure 5(a). The high polymer solution is placed in the injector. Under the action of a strong electric field, the solution forms a so-called Taylor cone at the nozzle position. A bunch of polymer stream is ejected from the cone tip and 


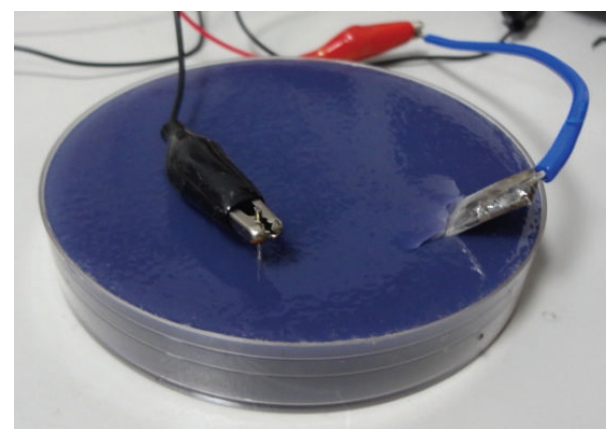

FIgURE 4: Preparing silver iodide by the conductivity method.

sprayed on the fiber collector in a helical whipping action under the attraction of the electric field. During ejection, the electric field aligns the polymer molecules into a fiber yarn within one microsecond. As the fiber filament is pulled and extended, its diameter is one thousandth of the fiber produced by the general micrometer nozzle, and the diameter of the end product is about $150 \mathrm{~nm}$. The fiber made by this method has high moisture permeability, high porosity, and high specific surface area; thus, it is extensively used in filtration, tissue engineering, biomedicine, and wound dressing material $[22,23]$.

In order to prepare PVA nanofibrous membrane with a good bacteriostatic effect, ESDM is used to prepare the silver nanoparticle PVA solution, and the electrospinning machine sprays the silver nanoparticle PVA solution into PVA nanofibrous membrane. The principle of preparing the silver nanoparticle PVA solution is the same as the principle for preparing nanosilver colloid in DI water, that is, the preparation method stated in Section 2.1. The only difference is that the PVA solution, at a concentration of $10 \%$, is prepared to replace the DI water, and the silver nanoparticles can be dispersed in the PVA solution. Finally, the aforesaid silver nanoparticle PVA solution is placed in the electrospinning machine and sprayed into the PVA nanofibrous membrane. Figure 5(b) shows the electrospinning machine used in this paper. The red frame is the main action part of the electrospinning machine. This paper uses UV-Vis to observe the characteristics of the PVA nanofibrous membrane and checks whether the silver nanoparticles are nucleated in PVA and wrapped in PVA.

\section{Results and Discussion}

3.1. Experiment on Bacteriostasis of Silver Iodide. In order to know the feasibility of using silver iodide in dressing, as prepared by the conductivity method, this paper used bacteriostasis experiments to observe the bacteriostatic effect of silver iodide and iodine. In order to avoid errors in the experimental results, the experiment was conducted twice, and the correctness of the first experiment was rechecked by the result of the second experiment.

The molds were placed in the iodine agar medium for the first experiment, and mold growth in the culture medium was observed on Day 3, Day 6, and Day 10. First, equivalent molds
TABLE 2: Conductance silver iodide bacteriostasis experiment (unit $\mathrm{cm})$.

\begin{tabular}{|c|c|c|c|c|c|c|c|}
\hline \multirow{3}{*}{$\begin{array}{l}\text { Culture } \\
\text { medium }\end{array}$} & \multicolumn{7}{|c|}{ Day } \\
\hline & \multicolumn{4}{|c|}{ 1st experiment } & \multicolumn{3}{|c|}{ 2nd experiment } \\
\hline & Day 1 & Day 3 & Day 6 & Day 10 & Day 1 & Day 6 & Day 10 \\
\hline Agar part & 0 & 2 & 4 & 6 & 0 & 2 & 4.5 \\
\hline $\begin{array}{l}\text { Povidone } \\
\text { iodine part }\end{array}$ & 0 & 0 & 1.2 & 2.8 & 0 & 0 & 2.2 \\
\hline $\begin{array}{l}\text { Silver iodide } \\
\text { part }\end{array}$ & 0 & 0 & 0 & 2.8 & 0 & 0 & 2 \\
\hline
\end{tabular}

are placed in the lower right part, lower left part, and upper part of the iodine agar medium, as shown in Figure 6(a). The orifice in the lower right part resulted from the removal of the silver wire electrode. As the silver wire electrode is connected to the cathode of the power supply, the negatively charged iodine ions are repelled by the cathodic electrons, and this part of the culture medium is white and free of iodine ion. The orifice in the lower left part resulted from the removal of the silver sheet electrode. As the silver sheet electrode is connected to the anode of the power supply, the iodine ions and silver ions can be combined into silver iodide. The mold growth in the culture medium on Day 3, Day 6, and Day 10 is as shown in Figures 6(b), 6(c), and 6(d), respectively. Figure 6 (b) shows that the molds breed only in the position without iodine ion. Figure 6(c) shows that the iodine in the culture medium is all dissipated after 6 days; thus, the culture medium is white. The lower left part of the culture medium is silver iodide; thus, it is light yellow. The mold begins to grow in the upper part with iodine. Figure 6(d) shows that while the mold has grown in the lower left part with silver iodide, the mold is less than that of the other two parts.

The locations of mold in the culture medium for the second experiment are the same as the first experiment, as shown in Figure 7(a). The mold growth in the culture mediums on Day 6 and Day 10 is as shown in Figures 7(b) and 7 (c), respectively. The experimental results show that the mold grew fastest in the position free of iodine and then in the position with iodine, followed by the mold growing most slowly in the position with silver iodide. This result matches the result of Experiment 1.

In the two experiments on the bacteriostasis of the agar medium, the growths of the molds in various parts of the culture mediums are as shown in Table 2 and Figure 8 . According to the results of two repeated experiments, the silver ions from conductance and the iodine ions can be combined into silver iodide. The experimental results show that iodine can actually inhibit the fungal growth; however, the silver iodide has better bacteriostatic effect. This conductivity method can be used for preparing povidone iodine in the future, meaning that the povidone iodine solution containing silver ions can enhance bacteriostatic ability.

3.2. Silver Nanoparticle PVA Nanofibrous Membrane Characteristic Analysis. In order to know the characteristics of 


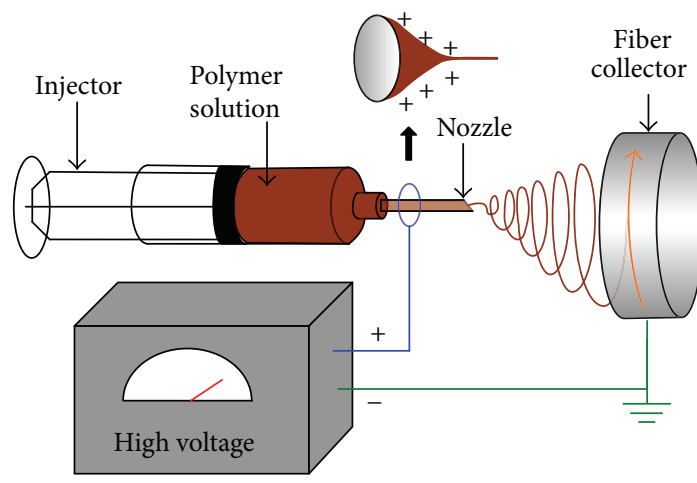

(a)

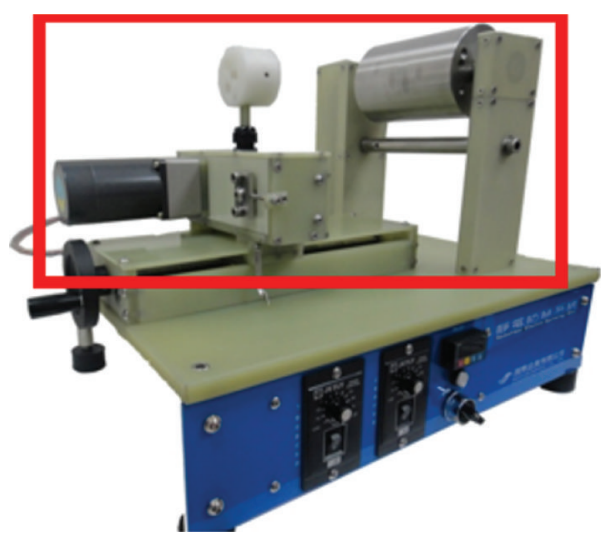

(b)

FIgURE 5: (a) Schematic diagram of electrospinning apparatus and (b) electrospinning machine.

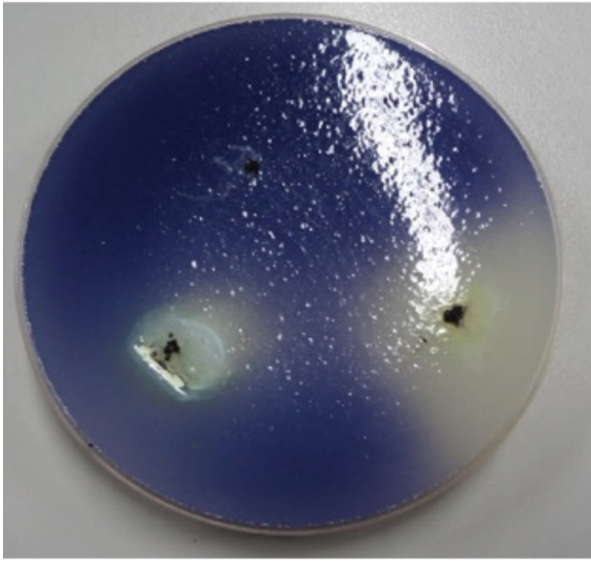

(a)

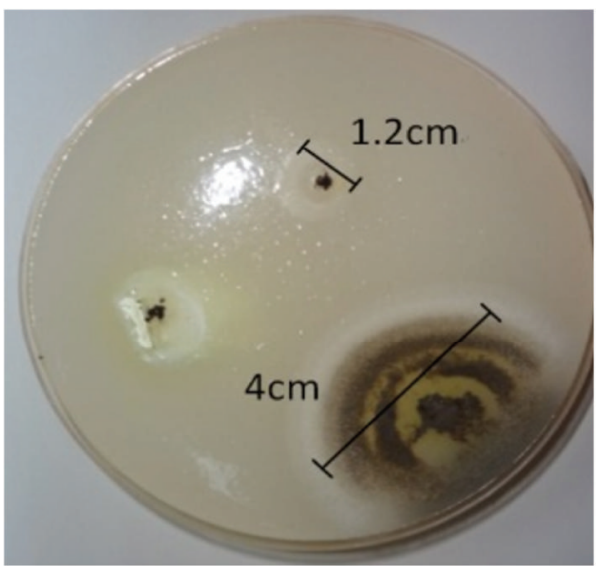

(c)

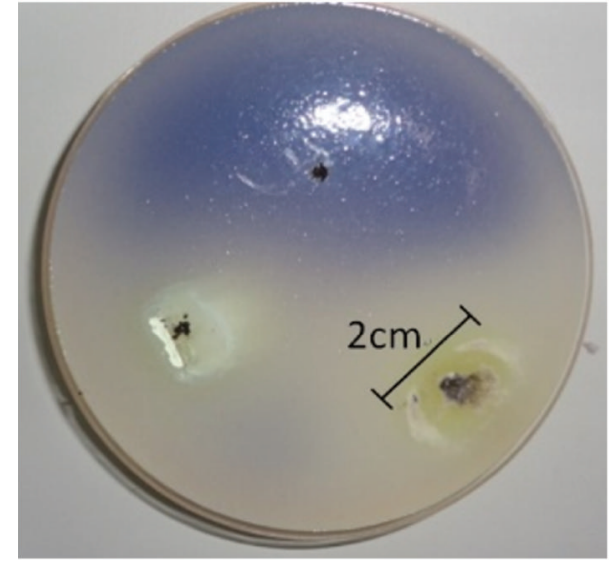

(b)

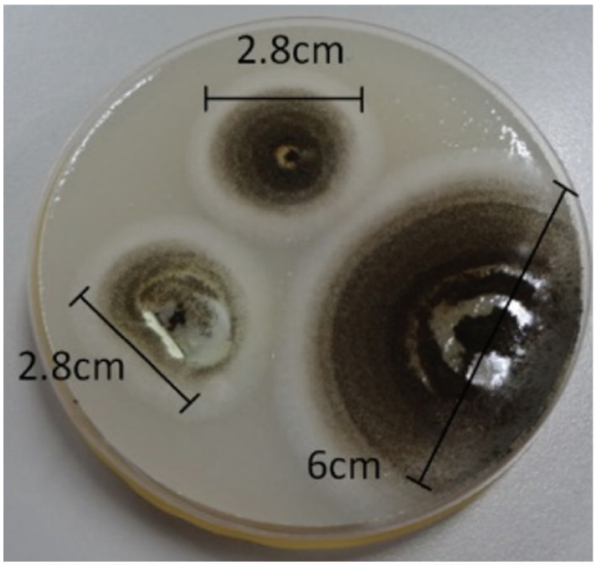

(d)

FIGURE 6: Silver iodide from conductance for the first time of (a) placement of molds, (b) 3 days after placement of molds, (c) 6 days after placement of molds, and (d) 10 days after placement of molds.

the PVA solution of nanosilver colloid, the absorption spectrum is observed by UV-Vis. The absorption spectrum of nanosilver colloid of DI water, as shown in Figure 3(a), is compared with the absorption spectrum of PVA solution of nanosilver colloid, as shown in Figure 9. The wavelength of the PVA solution nanosilver colloid is about $420 \mathrm{~nm}$, the wavelength of nanosilver colloid of DI water is about $410 \mathrm{~nm}$, and the difference is $10 \mathrm{~nm}$, which is the red shift. 


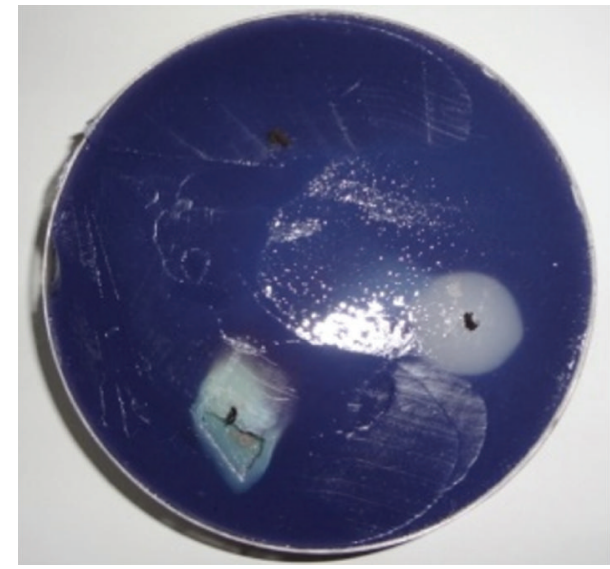

(a)

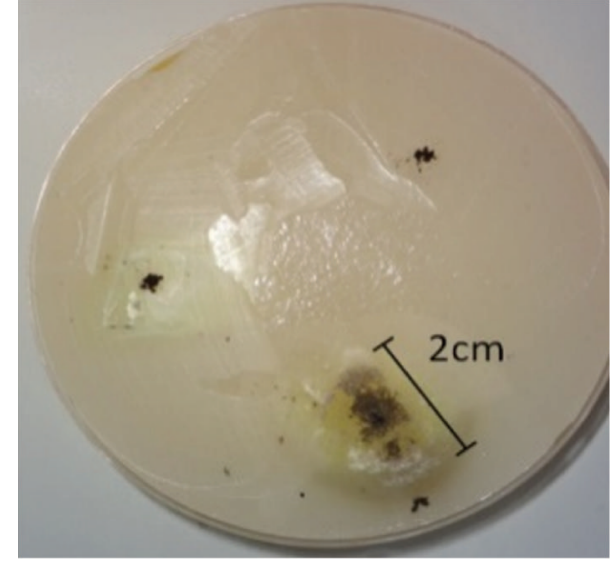

(b)

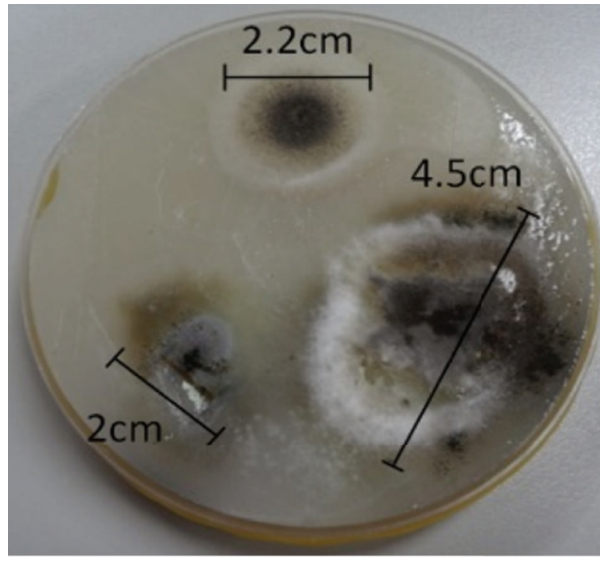

(c)

Figure 7: Silver iodide from conductance in (a) placement of molds, (b) 6 days after placement of molds, and (c) 10 days after placement of molds.

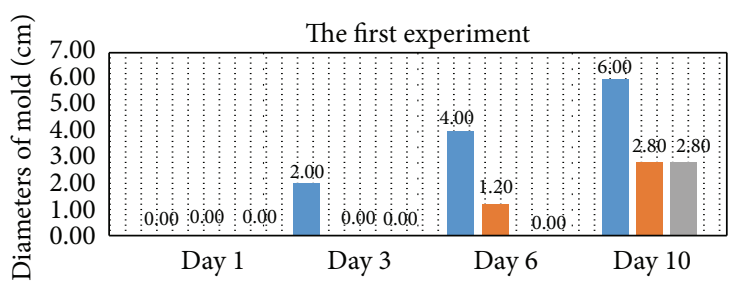

(Days)

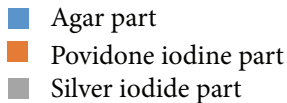

(a)

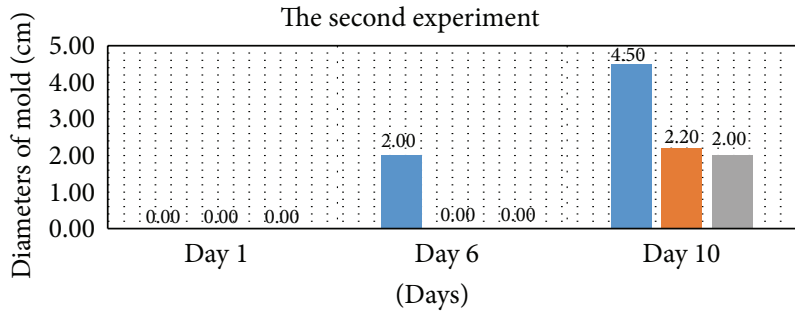

Agar part

Povidone iodine part

Silver iodide part

(b)

FiguRE 8: Bar graphs of conductance silver iodide bacteriostasis experiment for (a) result of the first experiment and (b) result of the second experiment.

Therefore, the PVA solution material is silver nanoparticle, and its absorption value is larger than the silver nanoparticles in DI water, meaning its concentration is higher than the silver nanoparticles in DI water.

The nanofibrous membrane, as prepared by the electrospinning machine, is light yellow, as shown in Figure 10. In order to further know the absorption peak of this nanofibrous membrane and review whether this nanofibrous membrane has nucleate silver nanoparticles, the nanofibrous membrane is affixed to a quartz sample cell, as shown in Figure 11. The UV-Vis analysis result of the membrane affixed to the quartz sample cell is as shown in Figure 12. The spectrogram shows 


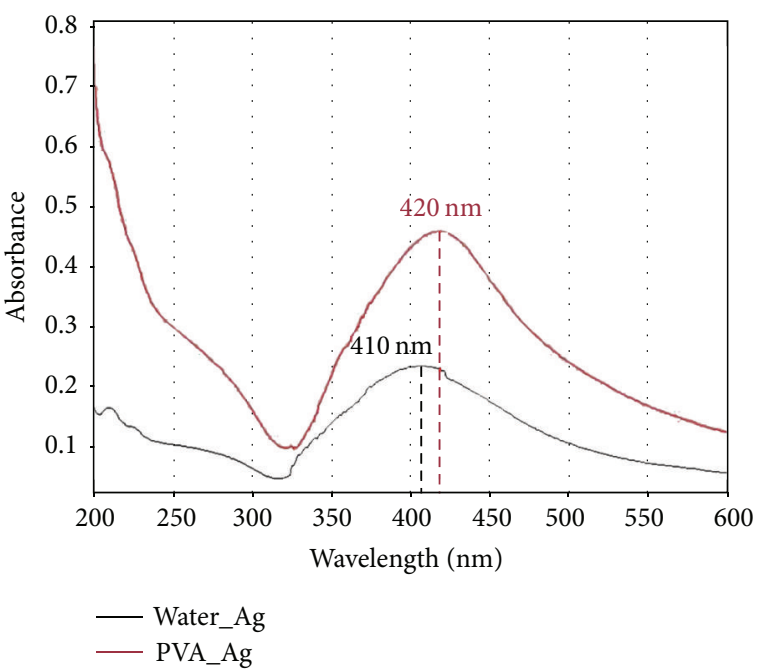

FIgURE 9: UV absorption spectrogram of water phase silver nanoparticle and PVA silver nanoparticle.

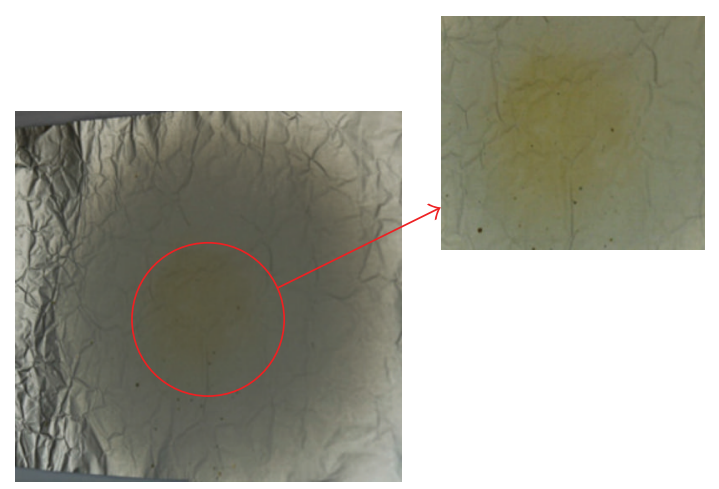

FIGURE 10: PVA electrostatic spun yarn with nanosilver colloid.

that the nanofibrous membrane has an approximate absorption peak of $415 \mathrm{~nm}$, which is approximately identical to silver nanoparticles. Therefore, this membrane does actually contain nucleate silver nanoparticles. As this membrane contains nanosilver colloid, it has sterilizing effect, and the PVA is innoxious and not harmful to the human body. Therefore, the nanofibrous membrane can be used in antiseptic dressing, gas mask membrane filters, and so forth.

3.3. The Application of Combining the Silver Iodide and Silver Nanoparticle PVA Nanofibrous Membrane. Due to the fact that the characteristics of silver iodide and silver nanoparticle PVA nanofibrous membrane are separately suitable for bacteriostasis, sterilization, and moist environment for the wound, this study combined them to a brand new wound care. The schematic diagram of the combined method is shown in Figure 13. First, place the iodine agar on the wounds and conduct the silver iodide into the possibly infective part. When the silver iodide touched the wounds, it will make the pathogen dead which is the purpose of sterilization. Then the silver nanoparticle PVA nanofibrous membranes cover the silver iodide. The fiber of the membrane can maintain the

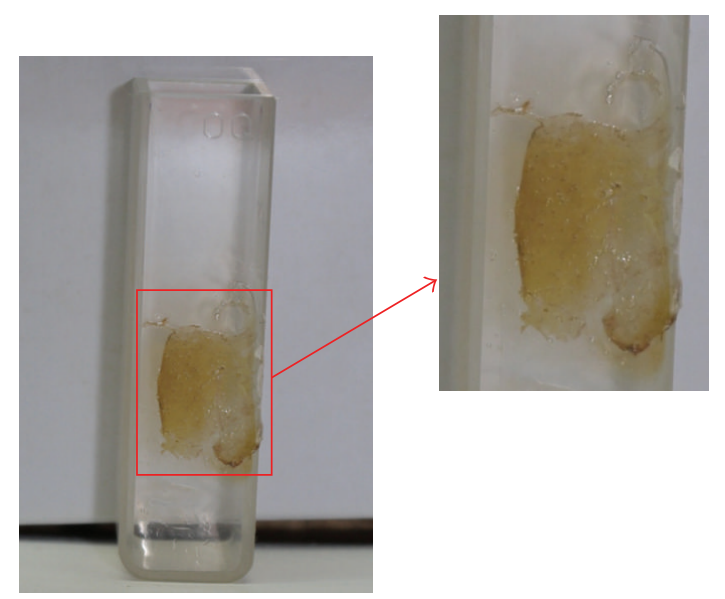

FIGURE 11: Quartz tube attached with PVA yarn with nanosilver colloid.

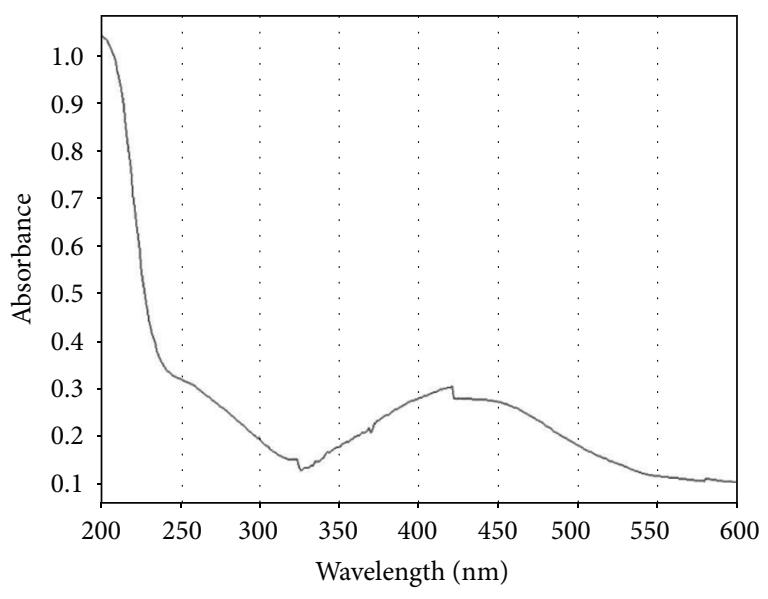

FIGURE 12: UV-Vis of PVA yarn with nanosilver colloid.

moist of healing environment and absorb most of the tissue fluid. When the two things made contact, the membrane will release the nanosilver particles which will suppress the growth of the bacterial and the generation of the odor.

\section{Conclusions}

As silver has strong bacteriostatic ability, and the bacteria will not become drug-fast, silver and silver nanoparticles are applicable to dressing. This paper uses the conductivity method, electric spark method, and the electrospinning technique to research a better silver-bearing dressing preparation method. This preparation process is free from chemical substances; thus, it matches the method of green energysaving processes, as prepared silver particles are cleaner than that created by the chemical method, and they are safe for humans. The findings are described as follows.

(1) Silver ion is successfully ionized from silver electrodes by using the conductivity method, which combines the iodine ion in the iodine agar medium into silver iodide. Multiple bacteriostasis experiments prove that 


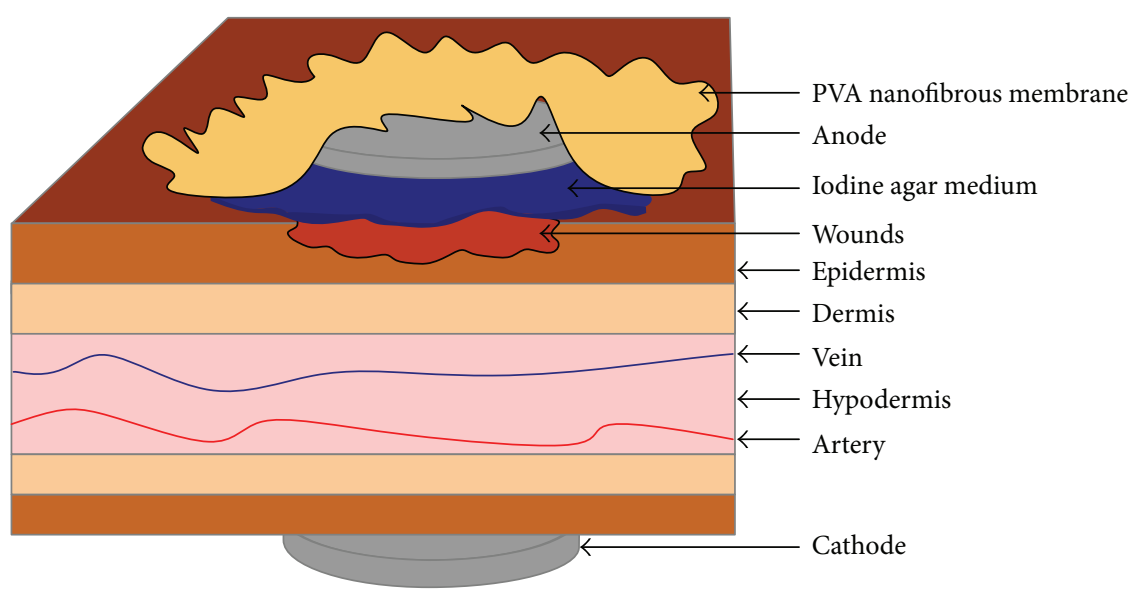

FIGURE 13: The schematic diagram of combining the silver iodide and silver nanoparticle PVA nanofibrous membrane.

silver iodide has a better bacteriostatic effect than povidone iodine. This conductivity method can be combined with the preparation of povidone iodine in the future, in order that the povidone iodine solution containing silver ions can enhance its bacteriostatic ability.

(2) Nanosilver colloid is successfully prepared in DI water by ESDM. According to the analysis results of UV-Vis, Zetasizer, and SEM-EDX, the principal component of this solution is silver, the wavelength of the nanosilver colloid is about $410 \mathrm{~nm}$, Zeta-Potential is about $-39.3 \mathrm{mV}$, and particle size is about $20 \mathrm{~nm}$. These data show that the solution actually does contain nanoscale silver colloid with good suspension stability.

(3) Nanosilver colloid is successfully prepared in PVA solution by ESDM. The wavelength of this nanosilver colloid is about $420 \mathrm{~nm}$, and the absorption value is larger than the silver nanoparticle in DI water, meaning the concentration of nanosilver colloid is higher than the nanosilver colloid in DI water.

(4) The light yellow silver nanoparticle PVA nanofibrous membrane is successfully prepared by ESDM and the electrospinning technique. The UV-Vis analysis result shows that the absorption peak of this membrane is about $415 \mathrm{~nm}$, which is enough to prove that the silver nanoparticles have been wrapped in a PVA nanofibrous membrane. Therefore, this nanofiber has good antibacterial ability and is suitable for antiseptic dressing.

(5) Combination of the silver iodide and silver nanoparticle PVA nanofibrous membrane in the wound care will give effect of bacteriostasis and sterilization to the wounds. Also, it will provide a more suitable and faster healing environment.

\section{Competing Interests}

The authors declare that there is no conflict of interests regarding the publication of this paper.

\section{Acknowledgments}

The authors would like to thank the Ministry of Science and Technology, China (MOST 103-2221-E-027-070-), for financially supporting this research.

\section{References}

[1] D. L. Monnet, F. M. MacKenzie, J. M. López-Lozano et al., "Antimicrobial drug use and methicillin-resistant Staphylococcus aureus, Aberdeen, 1996-2000," Emerging Infectious Diseases, vol. 10, no. 8, pp. 1432-1441, 2004.

[2] T. D. Hollingsworth, N. M. Ferguson, and R. M. Anderson, "Will travel restrictions control the international spread of pandemic influenza?" Nature Medicine, vol. 12, no. 5, pp. 497499, 2006.

[3] S. Srinivasan, P. T. Sudheesh Kumar, S. V. Nair, S. V. Nair, K. P. Chennazhi, and R. Jayakumar, "Antibacterial and bioactive $\alpha$ and $\beta$-chitin hydrogel/ nanobioactive glass ceramic/nano silver composite scaffolds for periodontal regeneration," Journal of Biomedical Nanotechnology, vol. 9, no. 11, pp. 1803-1816, 2013.

[4] A. Oya, S. Yoshida, Y. Abe, T. Iizuka, and N. Makiyama, "Antibacterial activated carbon fiber derived from phenolic resin containing silver nitrate," Carbon, vol. 31, no. 1, pp. 71-73, 1993.

[5] H.-K. Lee, M.-J. Shim, J.-S. Lee, and S.-W. Kim, "Characteristics of CO gas adsorption on modified natural zeolite," Materials Chemistry and Physics, vol. 44, no. 1, pp. 79-84, 1996.

[6] R. E. Burrell, J. P. Heggers, G. J. Davis, and J. B. Wright, "Efficacy of silver-coated dressings as bacterial barriers in a rodent burn sepsis model," Wounds, vol. 11, no. 4, pp. 64-71, 1999.

[7] S.-F. Lo, M. Hayter, C.-J. Chang, W.-Y. Hu, and L.-L. Lee, "A systematic review of silver-releasing dressings in the management of infected chronic wounds," Journal of Clinical Nursing, vol. 17, no. 15, pp. 1973-1985, 2008. 
[8] J. B. Wright, K. Lam, D. Hansen, and R. E. Burrell, "Efficacy of topical silver against fungal burn wound pathogens," American Journal of Infection Control, vol. 27, no. 4, pp. 344-350, 1999.

[9] I. Sondi and B. Salopek-Sondi, "Silver nanoparticles as antimicrobial agent: a case study on E. coli as a model for Gramnegative bacteria," Journal of Colloid and Interface Science, vol. 275, no. 1, pp. 177-182, 2004.

[10] J.-P. Guggenbichler, M. Böswald, S. Lugauer, and T. Krall, "A new technology of microdispersed silver in polyurethane induces antimicrobial activity in central venous catheters," Infection, vol. 27, no. 1, pp. S16-S23, 1999.

[11] H.-J. Jeon, S.-C. Yi, and S.-G. Oh, "Preparation and antibacterial effects of $\mathrm{Ag}-\mathrm{SiO}_{2}$ thin films by sol-gel method," Biomaterials, vol. 24, no. 27, pp. 4921-4928, 2003.

[12] J. A. Matthews, G. E. Wnek, D. G. Simpson, and G. L. Bowlin, "Electrospinning of collagen nanofibers," Biomacromolecules, vol. 3, no. 2, pp. 232-238, 2002.

[13] C.-Y. Liao, K.-H. Tseng, and H.-S. Lin, "Preparation of metallic aluminum compound particles by submerged arc discharge method in aqueous media," Metallurgical and Materials Transactions B, vol. 44, no. 1, pp. 91-97, 2013.

[14] J.-K. Lung, J.-C. Huang, D.-C. Tien et al., "Preparation of gold nanoparticles by arc discharge in water," Journal of Alloys and Compounds, vol. 434-435, pp. 655-658, 2007.

[15] D.-C. Tien, K.-H. Tseng, C.-Y. Liao, J.-C. Huang, and T.T. Tsung, "Discovery of ionic silver in silver nanoparticle suspension fabricated by arc discharge method," Journal of Alloys and Compounds, vol. 463, no. 1-2, pp. 408-411, 2008.

[16] S. Tariq Jilani and P. C. Pandey, "Analysis and modelling of EDM parameters," Precision Engineering, vol. 4, no. 4, pp. 215-221, 1982.

[17] M. Gostimirovic, P. Kovac, M. Sekulic, and B. Skoric, "Influence of discharge energy on machining characteristics in EDM," Journal of Mechanical Science and Technology, vol. 26, no. 1, pp. 173-179, 2012.

[18] S. Son, H. Lim, A. S. Kumar, and M. Rahman, "Influences of pulsed power condition on the machining properties in micro EDM," Journal of Materials Processing Technology, vol. 190, no. 1-3, pp. 73-76, 2007.

[19] K. H. Tseng, Y.-S. Kao, and C.-Y. Chang, "Development and implementation of a micro-electric discharge machine: realtime monitoring system of fabrication of nanosilver colloid," Journal of Cluster Science, vol. 27, no. 2, pp. 763-773, 2016.

[20] K.-H. Tseng, J.-L. Chiu, H.-L. Lee, Y.-S. Kao, and D.-C. Tien, "Spark parameter monitoring feedback system for preparation of nanosilver colloid in EDM," Materials and Manufacturing Processes, vol. 31, no. 2, pp. 186-193, 2016.

[21] D.-C. Tien, C.-Y. Liao, J.-C. Huang et al., "Novel technique for preparing a nano-silver water suspension by the arc-discharge method," Reviews on Advanced Materials Science, vol. 18, no. 8, pp. 750-756, 2008.

[22] Z.-M. Huang, Y.-Z. Zhang, M. Kotaki, and S. Ramakrishna, "A review on polymer nanofibers by electrospinning and their applications in nanocomposites," Composites Science and Technology, vol. 63, no. 15, pp. 2223-2253, 2003.

[23] K. H. Hong, "Preparation and properties of electrospun poly (vinyl alcohol)/silver fiber web as wound dressings," Polymer Engineering \& Science, vol. 47, no. 1, pp. 43-49, 2007. 

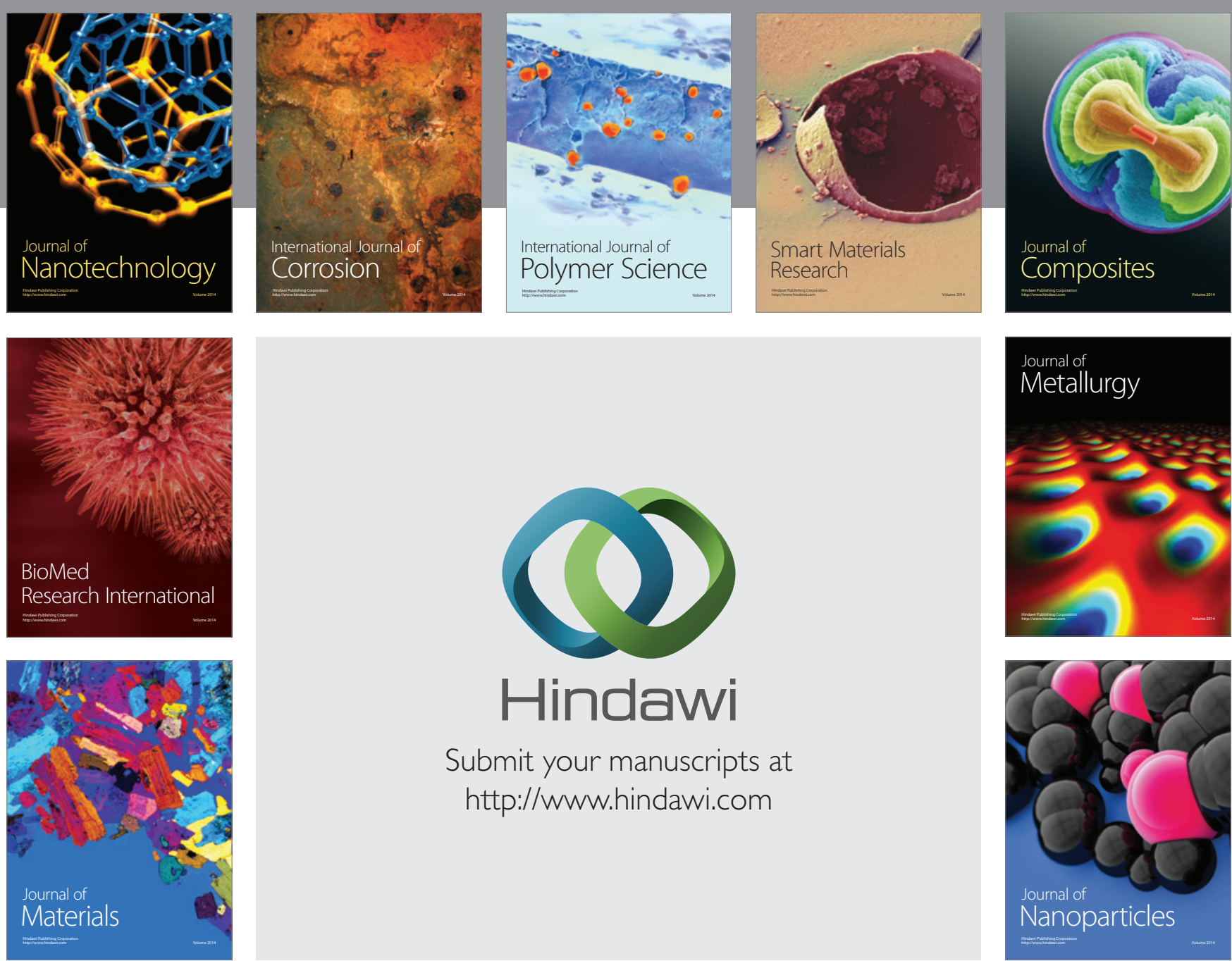

\section{Hindawi}

Submit your manuscripts at

http://www.hindawi.com

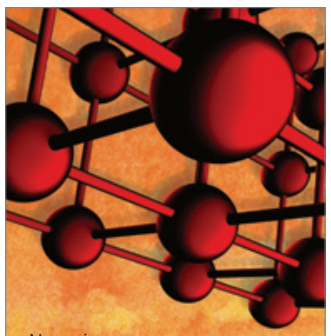

Materials Science and Engineering
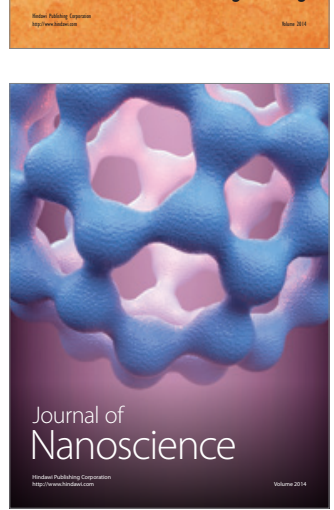
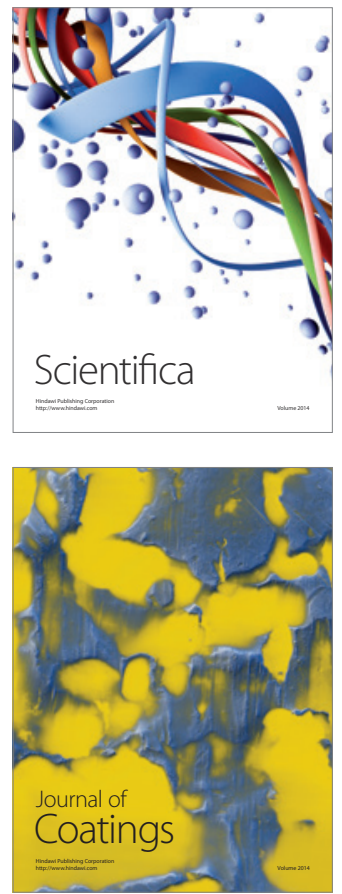
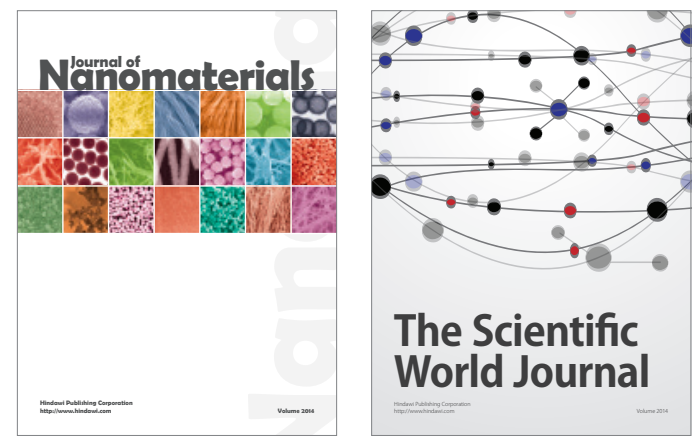

The Scientific World Journal
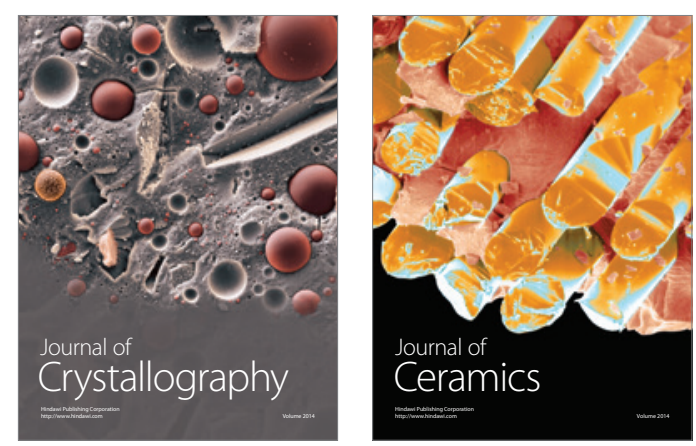
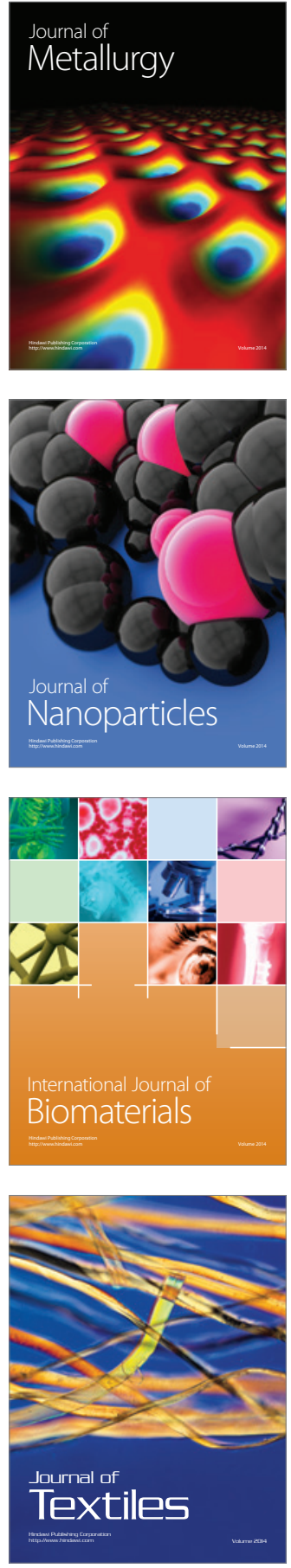